\title{
An Efficient K-SVD Algorithm of Dictionary Learning for HRRP Targets Recognition
}

\author{
Kun $\mathrm{CHEN}^{1, \text { a }}$, Yuehua $\mathrm{LI}^{2, \mathrm{~b}}{ }^{*}$ and, Yilu MA ${ }^{3, \mathrm{c}}$ \\ ${ }^{1}$ School of Electronic and Optical Engineering, Nanjing University of Science and Technology, \\ Nanjing, China \\ ${ }^{2}$ School of Electronic and Optical Engineering, Nanjing University of Science and Technology, \\ Nanjing, China \\ ${ }^{3}$ School of Electronic and Optical Engineering, Nanjing University of Science and Technology, \\ Nanjing, China

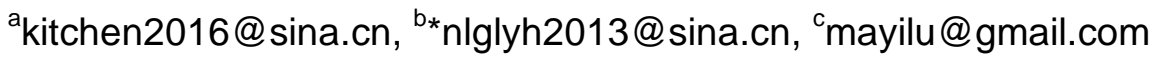

Keywords: sparse classification, K-SVD, dictionary learning, high resolution range profile.

Abstract. Inspired by the characteristics of sparse representation, we consider to recognize three military targets. To overcome the target-aspect sensitivity in radar high resolution range profile (HRRP), an improved dictionary learning algorithm, called auto-optimized fast K-SVD (AOF-KSVD), is proposed in this paper. We introduce the correlation threshold and effectiveness threshold into the K-SVD first, and then use the fast batch orthogonal matching pursuit method to update the atoms in the dictionary, which not only reduced the computation complexity, also the time of dictionary learning. Finally, the experiments result validated the performance of the proposed method.

\section{Introduction}

Radar HRRP recognition in the military applications is of great significance. So far, an enormous volume of literature has been devoted to investigate various radar target recognition methods and these methods can be roughly divide into three categories: the range profile based, especially the high resolution range profile (HRRP); the synthetic aperture radar (SAR) image based and the signal feature based. We mainly focus on the HRRP based radar target recognition in this paper. There are three challenges we will meet when use the HRRP to classify target: The target-aspect sensitivity, the time-shift sensitivity and the amplitude-scale sensitivity. The scattering center model [1], coherent averaging [2], Bayesian Gmma model [3] and HMM model [4] have been well studied to surmount the target-aspect sensitivity. For the time-shift sensitivity, the HRRP-based statistical model, the higher order spectra and the invariant feature have been proposed in [5,6,7]. Du et al. [5] use $l_{2}$-normalization of HRRP to overcome the amplitude-scale sensitivity.

Recently, some new methods integrated with the theory of sparse representation (SR) have been proposed, Wright et al. [8] first introduced the SR method into face recognition and it shown state-of-art performance. SR seeks a linear representation, with smallest number of no-zero elements, of the test sample in terms of the over complete dictionary and then be recovered efficiently via $l_{1}$-minimization. The SR method doesn't need the training process and assigns the class label to the class which has the least representation errors directly, so the SR can be view as a learning machine in which the classification process is accomplished by using reconstruction methods. As for radar target recognition, Dong et al. [9] proposed the joint sparse representation for SAR target recognition and Wang et al. [10] introduced the modified SR and manifold learning to the HRRP target recognition; they both achieved a certain success.

In this paper, we propose an improved dictionary learning algorithm for HRRP targets sparse recognition, called AOF-KSVD. We first introduce the correlation threshold coefficient and effectiveness threshold coefficient into the original K-SVD algorithm to constraint the number of atom in the dictionary, and then use the fast batch orthogonal matching pursuit method to solve the $l_{1}$-minimization problem. The experiments are executed to test the performance of the proposed 
method, from the two aspects of sparse representation accuracy and execution time, the results shown that our method is fast and effective.

\section{Sparse Classification and Dictionary Learning}

Sparse classification is based on the theory of SR, which is a task of reconstructing a signal by selecting a fewest bases from the over complete dictionary, meanwhile keeping the reconstruction error as small as possible. Give a training dictionary $D=\left[x_{1}, x_{2}, \ldots, x_{n}\right] \in R^{m \times n}$, for a test sample $y \in R^{m}$, the objective function of SR is to identify the smallest number of nonzero coefficients in $\alpha\left(l_{0}\right.$-norm minimization problem) and satisfy the equation $y=D \alpha$. The recent development of compressed sensing [11] shows that if the representation is sparse enough, the minimal solution is equivalent to the solution of following 11-norm minimization problem.

$$
\hat{\alpha}=\arg \min _{\alpha}\|\alpha\|_{1} \quad \text { s.t. } \quad \hat{y}=X \alpha
$$

when noise is present in the signal, a perfect reconstruction is typically not feasible. Therefore, we require that the reconstruction be within a error tolerance $\varepsilon>0$.

$$
\hat{\alpha}=\arg \min _{\alpha}\|\alpha\|_{1} \quad \text { s.t. } \quad\|\hat{y}-X \alpha\| \leq \varepsilon
$$

Often Eq.2 is approximated by loosening the error constraints and reconfigured to specifically include a regularization term as follows

$$
\hat{\alpha}=\arg \min _{\alpha}\left\{\|\hat{y}-X \alpha\|_{2}^{2}+\gamma\|\alpha\|_{1}\right\}
$$

where $\gamma$ is weighting constant, it gives a tradeoff between the reconstruction error and the sparsity of vector $\alpha$. The objective of the recognition is to find them smallest reconstruction error among all classes, given by

$$
\operatorname{identity}(y)=\arg \min _{i=1,2, \ldots, c}\left\|y-X^{i} \hat{\alpha}^{i}\right\|_{2}
$$

where $X^{i}$ and $\hat{\alpha}^{i}$ is the training samples and coding vector associated with the $i$-th class. From the discussion above, a fundamental question in the above formulation is the choice of the dictionary $D$. The dictionary can usually be determined from one of two sources: predesigned transform, such as Contourlet transform [12], or example data with adaptive training process. The K-SVD [13] algorithm is such a technique for training a dictionary from given samples. K-SVD algorithm accepts an initial over complete dictionary $D_{0}$, a number of iterations $k$, and a number of training samples arranged as the columns of the matrix $Y$. The algorithm aims to iteratively improve the dictionary to achieve sparse representations of the samples in $Y$, by solving the optimization problem

$$
\min _{D, A}\left\{\|Y-D A\|_{2}^{2}\right\} \quad \text { s.t. } \forall i,\left\|A_{i}\right\|_{0} \leq T_{0}
$$

where $A$ is the sparse coefficient matrix, $A=\left[\alpha_{1}, \alpha_{2}, \ldots, \alpha_{n}\right]^{T}$. The K-SVD algorithm involves two steps : Fix the current dictionary, get the sparse coefficient matrix $A$ through sparse-coded the samples in $Y$ and then fix the matrix $A$, update the dictionary atoms. The main innovation of K-SVD is in the latter step, which is accomplished while satisfy the constraint in Eq.5. To achieve this, the update step uses only the samples in $Y$ whose sparse coefficient use the current atom. Letting $I$ denote the indices of the signals in $X$ which use the $j$-th atom, the update is obtained by optimizing the target function over both the atom and its associated coefficient row in $A_{I}$. The resulting problem is a simple rank-1 approximation task given by

$$
\begin{aligned}
& \left\|Y_{I}-D A_{I}\right\|_{F}^{2} \\
& \left\{d^{\prime}, \alpha^{\prime}\right\}=\arg \min _{d^{\prime}, \alpha^{\prime}}\left\|E-d^{\prime} \alpha^{T T}\right\|_{F}^{2} \text { s.t. }\left\|d^{\prime}\right\|_{2}=1
\end{aligned}
$$

where $E=Y_{I}-\sum_{i \neq j} d_{i} A_{i, I}$ is the error matrix without the $\mathrm{j}$-th atom, $d^{\prime}$ is the updated atom, $\alpha$ 'is the new coefficients row in $A_{I}$. The problem can be solved directly via SVD decomposition. 


\section{The Proposed Method}

The K-SVD algorithm is quite computationally demanding, however, especially when the dimensions increase or the number of training samples becomes large. As target HRRP samples show a high correlation in a certain azimuth angle domain and the characteristics of target-aspect sensitivity. In order to make the dictionary atoms contain the samples in all different azimuth angles, we set a correlation threshold in the dictionary learning process, when the correlation coefficient is bigger than the presetting threshold, it removes the atoms automatically. On the other hand, basing on the entire sparsity contribution of one atom, we set a effectiveness threshold, when the sparse coefficient is lower than the presetting threshold, it removes the atoms automatically. Add suitable atoms to replace the removed atoms, so as to achieve the minimizing reconstruction error. Use the fast batch orthogonal matching pursuit algorithm [14] to update the dictionary. Combining with the characteristics of HRRP, we improve and optimize the K-SVD algorithm from the following three aspects.

1) Basing on the sparsity contribution of the atoms, set the effectiveness threshold $\gamma$,

2) In the optimal dictionary, any two atoms are orthogonal to each other, in order to make the dictionary achieve optimal, which require the correlation between the atoms as small as possible. Setting the correlation threshold $\sigma$, when the correlation coefficient between atoms is higher than the value, remove one and keep the other.

3) In Eq.7, we need to explicitly compute the matrix $E$, this computation is both time and memory consuming, as the size of $E$ is proportional to the number of training samples. In this paper, we use the fast batch orthogonal matching pursuit algorithm in [14] to update the atoms; it is an efficient implementation of dictionary learning, which reduces its complexity as well as its memory requirements.

We named the new algorithm auto optimized fast K-SVD (AOF-KSVD), The complete AOF-KSVD algorithm is given in Table 1.

Table 1 The AOF-KSVD Algorithm

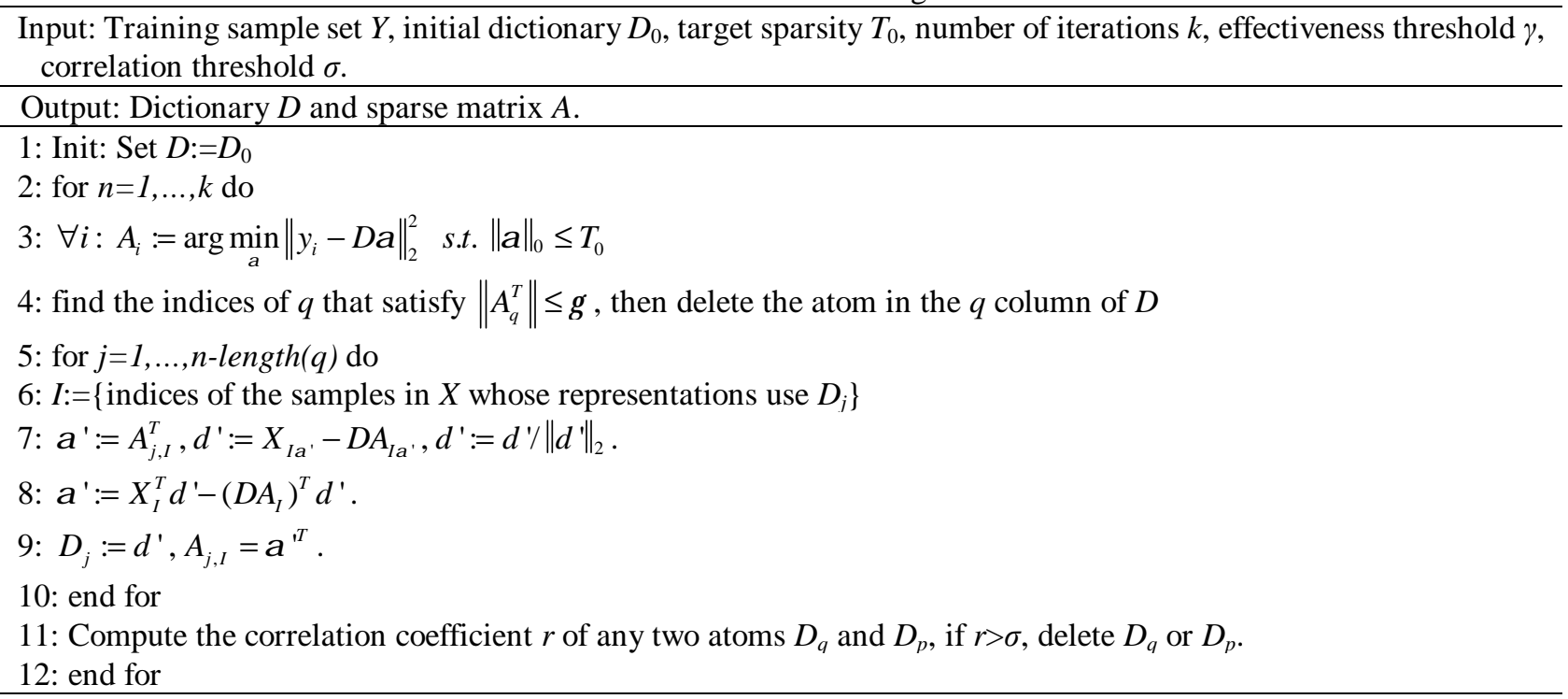

\section{Experiments}

In this section, we conducted experiment to evaluate the performance of the proposed AOF-KSVD algorithm. The purpose of the experiment is to compare the performance of different dictionary algorithms on three target HRRP datasets. in order to show the effectiveness of the improved dictionary learning algorithm, we compare the AOF-KSVD with the RLS_DLA [15], K-SVD [13], MOD [16] and ILS_DLA [17] algorithms. The ILS-DLA algorithm includes a forgetting factor $0 \leq \lambda_{i} \leq 1$, this opens the possibility of letting the training set $X$ be infinitely large. When $\lambda_{i}=0$, it reduces to the original MOD method; when $\lambda_{i}=1$, it's equivalent to the RLS-DLA; if without the forgetting factor $\lambda_{i}$, it is almost equivalent to the online dictionary learning (ODL) proposed in [18]. In this paper 
we set $\lambda_{i}=0.5$.

First, we fix the size of dictionary and its sparsity, comparing the sparse representation accuracy of dictionary learning algorithms. Here, we set the size of dictionary is 60 atoms, which is extracted from the three target HRRP datasets equivalently, set the sparsity $T_{0}=10$, then perform the dictionary learning algorithms and use the learning result to sparse representation all the samples in all three HRRP datasets. The whole experiments perform 20 times and get the average value as final result. We use the signal error ratio (SER) index to show the performance of the algorithms, it is the ratio between the signal power and reconstruction error and defined as following

$$
S E R=10 * \log 10 \cdot\left(\frac{\sum Y \cdot * Y}{\sum(Y-D A) *(Y-D A)}\right)
$$

where $*$ means the multiply of the corresponding elements in matrix.

For the AOF-KSVD, it has two input parameters: the correlation threshold $\sigma$ and effectiveness threshold $\gamma$. Figure. 1 shows the value of SER under different $\sigma$ and $\gamma$, we set the input parameter of AOF-KSVD as $\gamma=0.08, \sigma=0.28$ to obtain the max SER value.

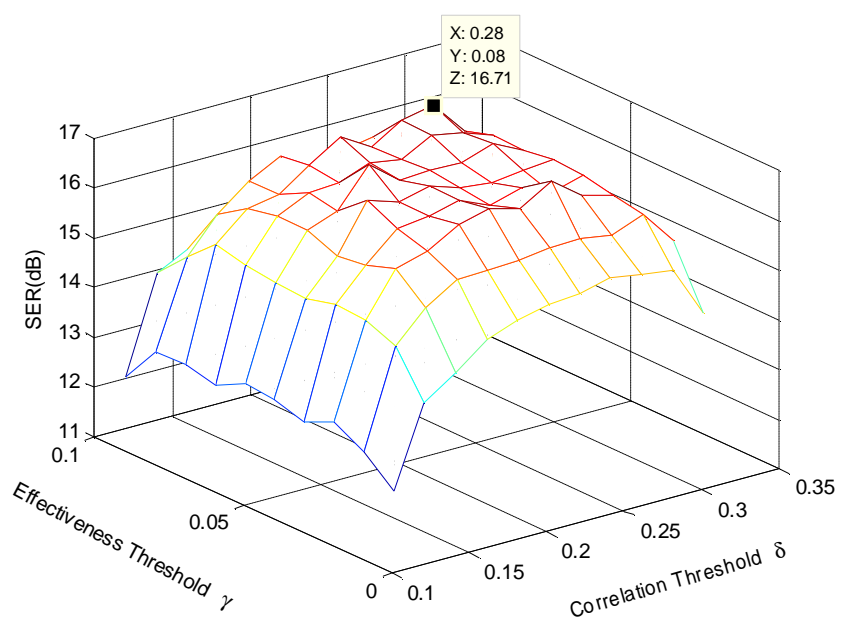

Figure.1 The SER under Different Input Parameters

Table 1 gives the SER of all the five algorithms, we can see that the SER, 16.8dB, of MOD is the highest, and $16.75 \mathrm{~dB}$ of the K-SVD is the next, our method is third, better than the ILS_DLA and RLS_DLA. The gap among them is very small, especially in the first three kinds of algorithms.

Table 1 The SER of All Comparing Algorithms

\begin{tabular}{|c|c|c|c|c|c|}
\hline Algorithm & ILS_DLA & K-SVD & RLS_DLA & MOD & AO-KSVD \\
\hline SER $(\mathrm{dB})$ & 16.44 & 16.75 & 16.29 & 16.80 & 16.71 \\
\hline
\end{tabular}

Second, we will test execution efficiency and calculation complexity of the algorithms. The execution time can direct reflect the execution efficiency and complexity of an algorithm. Here, we use the execution time to characterize the complexity and execution efficiency of an algorithm. Set the training set from 90 to 180 at interval 15 and the other settings remain the same. The time-consuming of all the methods on different sizes of training set are shown in Figure.2. It's clearly to see that the average time-consuming of RLS_DLA is the shortest, about 0.45 second, the time-consuming of AOF-KSVD and ILS_DLA almost equal, about 0.6 second and the three kinds of algorithm shows relatively time-consuming insensitive to the increase of training sample set. The K-SVD is also show time-consuming insensitive to the increase of training sample set, but the average time-consuming is about 6 seconds, much longer than the three above algorithms. The MOD algorithm, average over 10 seconds, is the most time-consuming and it sensitive to the increase of training sample set, as calculating the inverse of $X$ will takes much more time when the matrix become large. 


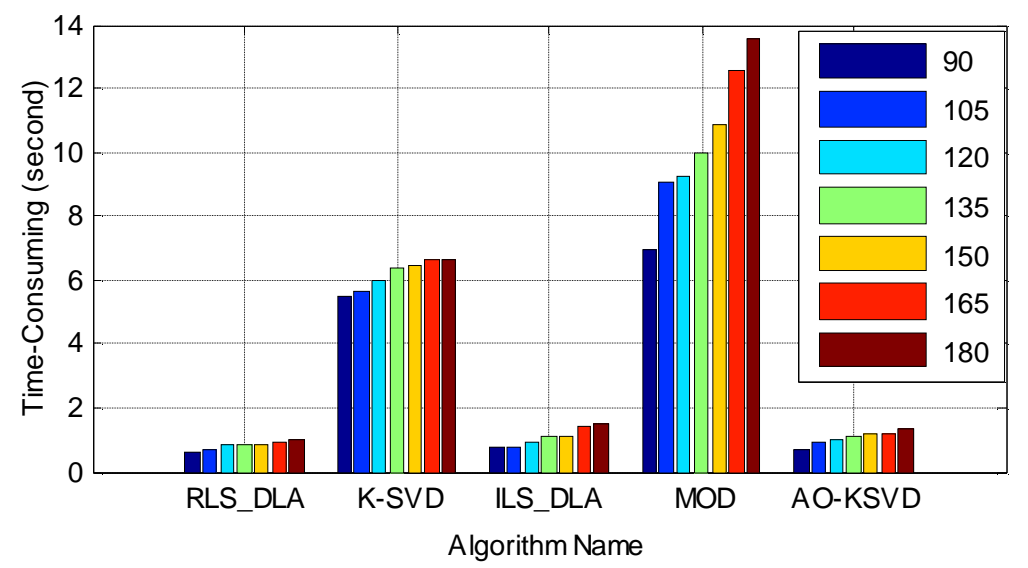

Figure 2 Time Consuming of All Algorithms under different Training Set

Taking both the representation performance and complexity into consideration, we can obtain the following conclusions: although ILS_DLA and RLS_DLA have less time-consuming, but they have poor representation performance, the representation performance of K-SVD and MOD is high, but they are time consuming. On the contrary, the AOF-KSVD algorithm not only has the proportional representation performance, but also has obvious advantage in execution efficiency and complexity. So the proposed AOF-KSVD algorithm is effective to the HRRP sparse representation problem.

\section{Conclusions}

In this paper, we proposed a modified fast dictionary learning method used in sparse representation, named AOF-KSVD, to accomplish the training phase of HRRP target sparse representation. In the training phase, we introduce the correlation coefficient and effectiveness coefficient to improved the original K-SVD algorithm. Experiments result shows that the AOF-KSVD algorithm not only has the proportional representation performance, but also has obvious advantage in execution efficiency and complexity.

\section{References}

[1] H.J. Li and S.H. Yang, Using range profiles as feature vectors to identify aerospace objects, IEEE Trans. Antennas and Propagation. vol.41, no.3, pp.261-268, 1993.

[2] A. Zyweck and R.E. Bogner, Coherent averaging of range profiles, IEEE International Radar Conference, pp.456-461,1995.

[3] K. Copsey and A. Webb, Bayesian gmma mixture model approach to radar target recognition, IEEE Trans. Aerosp. Electron. Syst., vol.39, no.4, pp.1201-1217, 2003.

[4] X.J. Liao, P. Runkle and L.Carin, Identification of ground targets from sequential high range resolution radar signatures, IEEE Aerosp. Electron. Syst., vol.38, no.4, pp.1230-1242, 2002.

[5] L. Du, H.W. Liu and Z. Bao, Radar HRRP statistical recognition: parametric mode and model selection, IEEE Trans. Singal Process., vol.56, no.5, pp.1931-1944, 2008.

[6] L. Du, H.W. Liu and Z. Bao, Radar HRRP target recognition based on higher order spectra, IEEE Trans. Singal Process., vol.53, no.7, pp.2359-2368, 2005.

[7] K. Kim, D. Seo and H. Kim, Efficient radar target recognition using the MUSIC algorithm and invariant feature, IEEE Trans. Antennas and Propagation. vol.50, no.3, pp.325-337, 2002.

[8] L.M. Novak, S.D. Halversen, G.J. Owirka and M. Hiett, Effects of polarization and resolution on the performance of a SAR automatic target recognition system, Lincoln Lab. J., vol.8, no.1, pp.49-68,1995. 
[9] G.G. Dong, G.Y. Kuang, N. Wang, L.J. Zhao and J. Lu, SAR target recognition via joint sparse representation of monogenic signal, IEEE joural of selected topics in applied earth observations and remote sensing., vol.8 no.7, pp.3316-3328, 2015.

[10] J.Q. Wang, Y.H. Li and K. Chen, Radar high-resolution range profile recognition via geodesic weighted sparse representation, IET Radar Sonar Navig., vol.9, no.1, pp.75-83, 2014.

[11] Candes E J, Romberg J K, Tao T. Stable signal recovery from incomplete and inaccurate measurements [J]. Communications on Pure \& Applied Mathematics, vol.59, no.8, pp.1207-1223, 2006.

[12] M. Do and M. Vetterli, "The contourlet transform: an efficient directional multiresolution image representation”, IEEE Transaction on Image Processing., vol.14, no.12, pp.2091-2106, 2005.

[13] M. Aharon, M. Elad and A. Bruckstein, K-SVD: an algorithm for designing overcomplete dictionaries for sparse representation, IEEE Transaction on Image Processing., vol.54, no.11, pp. 4311-4322, 2006.

[14] R. Rubinstein, M. Zibulevsky and M. Elad, Efficient implementation of the K-SVD algorithm using batch orthogonal matching pursuit, Cs Technical Reports, 40, 2008.

[15] K. Skretting and K. Engan, Recursive least squares dictionary learning algorithm, IEEE Transactions on Signal Processing., vol.58, no.2, pp.2121-130, 2010.

[16] K. Engan, S. O. Aase and J. H. Husey, Method of optimal directions for frame design, Proceeding of IEEE International Conference on Acoustics, Speech, \& Signal Processing, vol.5, pp.2443-446, 1999.

[17] K. Engan, K. Skretting and J. H.Husoy, Family of iterative LS-based dictionary learning algorithms, ILS-DLA, for sparse signal representation, Digital Signal Processing., vol.17, no.1, pp.32-49, 2007.

[18] J. Mairal, F. Bach, J. Ponce and G. Sapiro, Online dictionary learning for sparse coding, Proceeding of International Conference of Machine Learning (ICML), Montreal, Canada, pp.689-696, 2009. 